SERGE BAHUCHET

\title{
Les Pygmées d'aujourd'hui en Afrique centrale
}

Les Pygmées d'Afrique centrale sont connus comme une des dernières populations du monde à vivre encore de la chasse et de la cueillette. De nombreuses publications, dont plusieurs très célèbres, s'attachent à en décrire le mode de vie particulier. Le public les connaît aussi grâce à des films - dont quelquesuns sont excellents - qui visent à montrer l'adaptation culturelle de ces peuples à l'environnement forestier.

Cependant, l'image ainsi perpétuée ne correspond plus de nos jours à ce que le voyageur peut observer lorsqu'il traverse les régions forestières ; nombre de Pygmées présentent un mode de vie fort modifié. Par ailleurs, des pressions importantes s'exercent sur le milieu forestier lui-même, qui ne sont pas sans effet sur l'économie des populations qui y vivent, agriculteurs comme chasseurs-cueilleurs.

Après avoir passé de nombreuses années à étudier le mode de vie traditionnel de groupes de Pygmées Aka de République centrafricaine, il nous a paru intéressant et utile d'examiner les transformations qui se manifestent de nos jours dans les diverses régions forestières d'Afrique équatoriale. En effet, on remarquera qu'en dépit de la dispersion et des différences existantes entre les diverses populations pygmées, du Cameroun au Zaïre, on rencontre partout les mêmes types d'évolution du mode de vie. Aussi avons-nous tenté une systématisation de ces tendances, en cherchant à dégager leurs racines historiques et sociales ${ }^{1}$.

1. Les documents qui m'ont servi pour ce travail sont majoritairement inédits. J'ai en effet eu accès à de nombreux rapports régionaux, ainsi qu'à de nombreuses communications personnelles, par lettres ou orales, provenant de collègues et de personnes résidant et œuvrant pour le développement en Afrique équatoriale. Une première version de ce texte, écrite en 1986 a beaucoup circulé, j'ai tenu compte dans cette nouvelle version des informations complémentaires qui m'ont été fournies par mes correspondants. Principales sources non publiées :

- Cameroun : documents de l'Institut des Sciences humaines (professeur Loung, G. Nguima Mawoung 1981, 1982), rapports des Petites Sœurs de Jésus (Bipindi), documents du ministère des Affaires sociales (rapport Zumbach 1982, rapport Oyono); documents personnels (missions de 1983 à 1985) ;

- Centrafrique : rapport sur Belemboke (Coudrin) et documents personnels (missions de 1972 à 1985) ; - Congo : rapport de mission Orstom de H. Guillaume (1982) ;

- Zaïre : Twa, documents de H. Pagezy; Mbuti, documents personnels missions 1989 et 1990. Principales sources publiées : Althabe 1965, Bahuchet 1985, Bailey 1982, Coudrin 1988, Demesse 1978, Hart 1978, Hart \& Hart 1984, Loung 1959, Nguima-Mawoung 1981, Pagezy 1982, 1986, Peacock 1984, Schultz 1986, Sulzmann 1986, Turnbull 1983, Waehle 1986. 


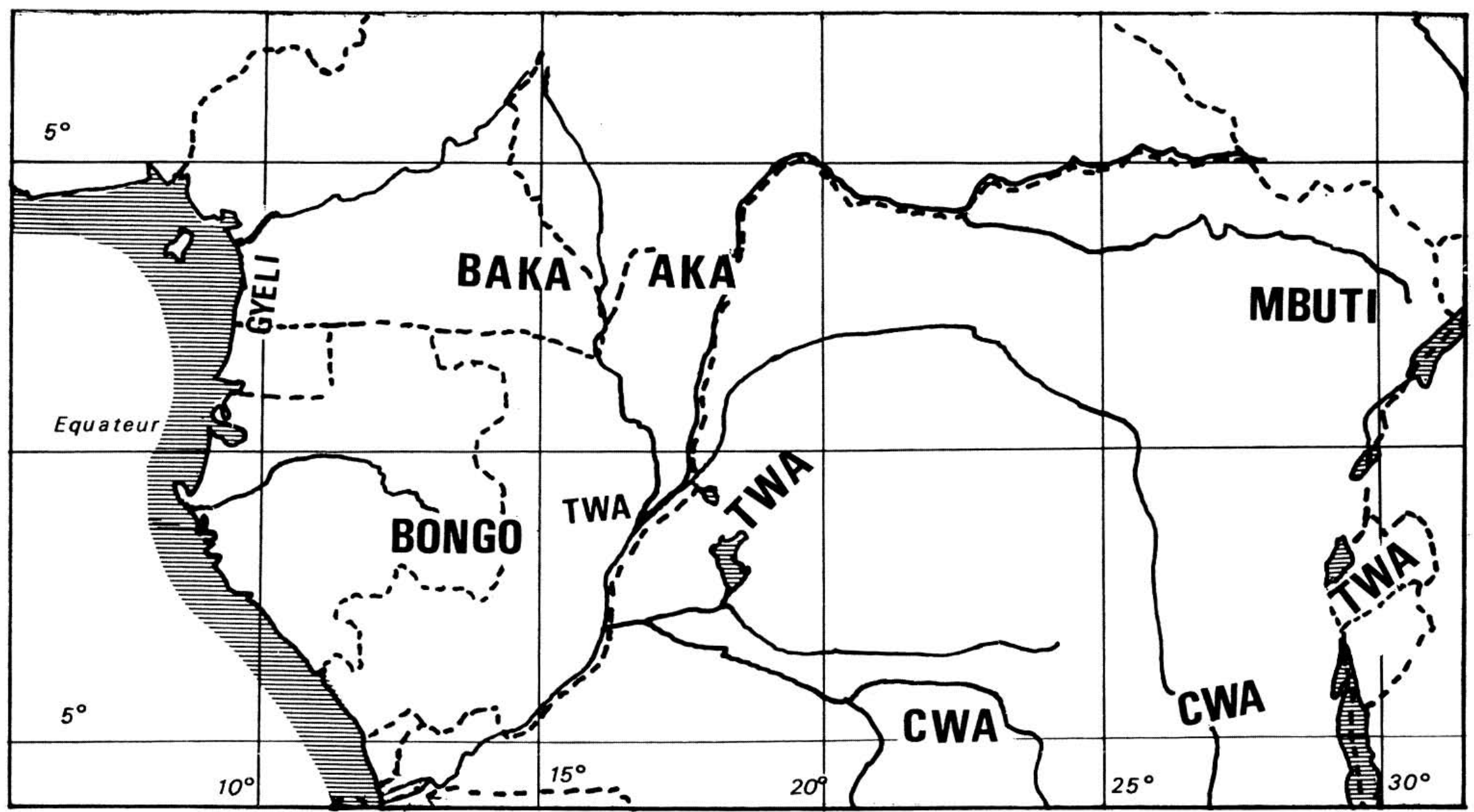

Principaux groupes pygmées d'Afrique 
En réalité, le terme de " Pygmées » réunit un peu artificiellement plusieurs groupes ethniques, différents au point de vue physique, linguistique et, dans une certaine mesure, culturel, et disséminés de la côte atlantique au Rwanda : BaMbuti $^{2}$ de l'est du Zaïre (forêt de l'Ituri) qui se divisent en au moins trois grands groupes, Efè, Asua et Mbuti proprement dits ; BaKola ou BaGyeli (sudouest du Cameroun), Baka et Bangombe (sud-est du Cameroun, nord du Gabon et nord-ouest du Congo), BaAka et BaMbènzèlè (République centrafricaine et nord du Congo), BaBongo ou Akoa (Gabon et ouest du Congo). Ces derniers groupes étaient connus sous le terme générique de BaBinga.

Un troisième groupe, important bien que morcelé et dispersé, est connu sous le nom de BaTwa ou BaCwa, et présent au Rwanda et au Burundi, ainsi qu'au sud-est et au centre-ouest du Zaïre (Kazadi 1981).

Les Mbuti, les Baka et les Aka ont une économie tout à fait comparable (chasse et collecte, semi-nomadisme, symbiose avec les agriculteurs), alors que les Twa sont aussi pour la plupart chasseurs-collecteurs, mais nettement plus sédentaires, et forment des castes au sein des sociétés d'agriculteurs ; certains Twa du Rwanda sont spécialisés dans la poterie.

La șituation économique et sociale actuelle des Pygmées dans la plupart des pays forestiers d'Afrique équatoriale se situe directement et sans rupture dans le prolongement de la situation bipartite qui a prévalu à travers les siècles : les relations entre les chasseurs-collecteurs Pygmées et les Grands Noirs agriculteurs sur brûlis. Pour cette raison, nous évoquerons succinctement l'histoire des relations entre Pygmées et villageois, puis présenterons le contexte économique actuel des forêts d'Afrique centrale, dans la mesure où ce sont les modifications du mode de vie des villageois qui influent notablement sur le mode de vie des Pygmées.

\section{MODE DE VIE TRADITIONNEL DES PYGMÉES}

\section{Techniques et subsistance}

Les divers groupes pygmées qui sont, en réalité, autant d'ethnies différentes, vivent selon une économie fondée sur la chasse et la collecte, c'est-à-dire basée sur l'exploitation des ressources naturelles, sans transformation du milieu par l'agriculture ou l'élevage. Le seul animal domestique est le chien.

Les sociétés pygmées sont caractérisées par l'absence de spécialisation, chaque membre de la communauté étant capable de fabriquer les objets dont il a besoin. Toutefois les Pygmées ne transforment ni le métal ni l'argile, obtenant par des échanges avec les sociétés voisines les ustensiles de première nécessité (marmites, couteaux, fers de hache et de sagaie).

Ils vivent en campement, dans des huttes végétales hémisphériques. Ces campements sont temporaires et durent rarement plus de quelques mois. Mobiles,

2. Suivant la convention habituelle en linguistique pour les noms des langues bantoues, nous ne mettrons plus dans la suite du texte les préfixes : Mbuti, au lieu de MoMbuti (singulier), BaMbuti (pluriel). 
les Pygmées ne sont nulle part nomades, car leurs déplacements s'effectuent toujours à l'intérieur d'une aire particulière de forêt, territoire restreint aux limites définissables. Dans tous les cas, une des extrémités de ce territoire est le village des agriculteurs avec lesquels les membres du camp effectuent leurs échanges.

Une grande simplicité de moyens caractérise la technologie : peu d'objets mais avec une large gamme d'emploi. L'approvisionnement est assuré grâce aux produits forestiers et c'est la quête alimentaire qui occupe la plus grande partie du temps. Les activités sont très souvent collectives mais elles ne sont jamais dirigées par un chef. Célèbres pour leurs prouesses à la chasse à l'éléphant, les Pygmées se nourrissent cependant surtout de mammifères plus communs, potamochères et céphalophes, ainsi que rongeurs géants (porcs-épics, rats de Gambie) et singes arboricoles.

Tous les groupes pygmées n'utilisent pas exactement les mêmes techniques, mais dans tous les cas le groupe connaît plusieurs types de capture qu'il emploie tour à tour, selon les saisons, les disponibilités de gibier et selon le nombre de chasseurs présents au même moment. Les Mbuti et les Asua du sud-est et de l'ouest de l'Ituri chassent collectivement avec des filets et des arcs, alors que les Efè du nord-est de l'Ituri chassent à l'arc sans les filets. Les Aka de RCA et du Congo chassent au filet avec des sagaies, tandis que les Baka n'utilisent que leurs sagaies. Mais Mbuti et Aka chassent aussi à la sagaie, en pistant les gros mammifères et tous les groupes tuent les rongeurs en les dénichant dans leur terrier, avec ou sans l'aide de chiens, et abattent les singes et les gros oiseaux avec des arcs ou des arbalètes, aux minces flèches empoisonnées, en bois de palmier. Certaines de ces chasses sont individuelles (arc ou arbalète), d'autres mobilisent tous les hommes d'un camp (sagaie) ou bien plusieurs campements (battues aux filets), cependant que deux ou trois personnes suffisent pour capturer les porcs-épics. Si les pistages à la sagaie sont toujours exclusivement effectués par les hommes, les femmes en groupe participent à la chasse aux filets, et souvent c'est le couple qui chasse les porcs-épics. Dans quelques ethnies (Kola, Aka), les femmes peuvent même chasser en groupe, si les hommes ne le font pas.

Les produits carnés sont complétés par des produits de collecte, animaux et végétaux : tubercules d'ignames sauvages, feuilles de lianes, champignons, noix oléagineuses, chenilles, termites et larves de coléoptères dans le bois mort. On récolte également le miel des abeilles sauvages. Les femmes et les jeunes filles assurent principalement la collecte, mais il est fréquent que les familles conjugales partent ensemble récolter des noix ou ramasser des chenilles, tout comme il est usuel que les hommes, au cours de leurs chasses, recueillent tout ce qu'ils rencontrent en chemin. Mais la seule activité qui soit entourée d'autant d'attention que la chasse est la récolte du miel, qui nécessite de localiser des ruches à plus de 30 mètres au-dessus du sol et de grimper à l'arbre, avec une ceinture de liane, pour extraire les rayons à la hache. Ce sont les hommes qui se chargent de cette récolte.

Les prises de chasse collectives font l'objet d'un partage entre les chasseurs ayant participé à l'encerclement et à l'abattage du gibier. Par contre, les produits de collecte, hormis le miel, ne sont pas systématiquement partagés mais seulement distribués en cas d'abondance. 
Les aliments sont rarement conservés. La viande peut être boucanée, généralement en vue d' " exportation » vers les villages; les chenilles sont séchées et peuvent être gardées quelques mois, de même que certaines graines oléagineuses. Mais ordinairement la récolte du jour est préparée et consommée dans les quarante-huit heures. La cuisson se fait à l'eau dans des marmites, ou bien à l'étouffée dans des emballages de feuilles disposés dans la braise ou suspendus au-dessus du foyer. Les plats préparés vont toujours par paire : d'une part une sauce qui contient la viande, les légumes (feuilles, champignons) et les condiments (graines et amandes, piment), d'autre part un féculent (igname, manioc ou banane plantain) qui constitue l'aliment de base. Ces plats sont partagés et largement distribués à l'intérieur du campement.

\section{Organisation sociale}

Dans toutes les ethnies pygmées, l'unité socio-économique est le campement. C'est à ce niveau que les activités collectives s'opèrent ; c'est aussi à ce niveau qu'ont lieu partages et distributions.

Le campement est généralement constitué d'une dizaine de huttes formant un groupe assez restreint (de 30 à 70 personnes). Ce groupe compte un certain nombre d'hommes étroitement apparentés (des frères ou des cousins) mais aussi des parents de leurs épouses, ainsi que des sœurs avec leur mari. C'est l'aîné (père, oncle ou frère le plus âgé) qui bénéficie de l'autorité morale.

Chaque groupe entretient avec les autres des relations nombreuses. Les groupes voisins se réunissent périodiquement, ce qui est l'occasion de grandes chasses collectives, mais aussi de nombreuses cérémonies et de danses rituelles. Les familles conjugales rendent souvent visite à leurs parents vivant dans d'autres camps, pour des durées allant de quelques jours à plusieurs mois. A ces occasions, les visiteurs participent à la vie quotidienne comme ils le font d'ordinaire dans leur campement d'origine. Cette pratique très générale rend la composition des campements toujours changeante, car il $\mathrm{y}$ a à tout moment une famille qui est en voyage, une autre qui vient en visite. Le choix des conjoints dans des camps éloignés, ainsi que la pratique du "service de mariage " (séjour de longue durée du marié dans la communauté de son épouse) favorisent les visites.

La mobilité des campements résulte d'une subtile combinaison de causes : appauvrissement des ressources alimentaires, mais aussi importance numérique du groupe, nécessité de visites, proximité de groupes voisins, et encore troubles sociaux ou décès. Au fil des mois, les communautés se regroupent ou se scindent alternativement, en un perpétuel mouvement de fusion et de fission.

La chasse pèse d'un poids déterminant dans l'organisation sociale. En premier lieu parce qu'elle est l'activité qui mobilise les forces des membres de la communauté, en second lieu car c'est autour d'elle que se cristallisent les étapes du cycle de développement des individus ainsi qu'une partie des activités religieuses. En effet, on observe une étroite interdépendance des capacités de chasseur des jeunes gens, de leur aptitude au mariage et de leur participation aux grandes chasses aux mammifères prestigieux (éléphant). Plusieurs rituels encadrent les activités de chasse, propitiatoire aussi bien qu'expiatoires. La seconde activité d'importance est la récolte du miel, pourvu d'une haute valeur 
symbolique car considéré comme liqueur de vie. La récolte du premier miel de la saison est précédée de rituels collectifs (seul cas pour une activité relevant de la collecte) cependant que la saison du miel est, chez les Mbuti du Zaïre, marquée par une dispersion temporaire des groupes (Ichikawa 1981).

L'organisation religieuse des trois groupes qui nous sont le mieux connus présente des similitudes (Bahuchet et Thomas 1987) : Dieu créa le monde, c'està-dire la forêt, puis ayant créé les premiers couples (incestueux), il se désintéressa des affaires du monde. Un Esprit suprême, dieu de la Forêt, agit à sa place, régnant sur le monde des mânes, médiateurs des humains vivants. Il est le maître des ressources qu'il procure aux hommes. Les rituels sont liés à l'incertitude économique ; ils concernent principalement la chasse mais jamais la collecte (ni végétaux, ni insectes). Les cérémonies les plus importantes sont celles qui suivent les levées de deuil et l'implantation d'un nouveau campement : ce sont de grandes manifestations très sacrées, réunissant un grand concours de population aux cours desquelles l'Esprit intervient lui-même (soit par un masque de raphia, soit par des sons de trompes). Ces rituels sont toujours d'une grande importance dans l'organisation socio-économique car ils marquent la réaffirmation de la communauté autour de son dieu après une crise grave.

\section{PYGMÉES ET GRANDS NOIRS : UNE LONGUE HISTOIRE COMMUNE...}

Les relations des Pygmées avec les peuples de Grands Noirs, qui vivent dans la même forêt équatoriale, ne cessent de poser problème aux observateurs étrangers, qui ont du mal à les interpréter ainsi qu'à évaluer leurs fondements. Néanmoins c'est précisément là que réside le trait le plus important pour l'analyse de l'évolution du mode de vie des Pygmées. On constatera, en effet, que les modifications s'effectuent toujours par rapport à ces relations, soit que l'évolution de l'économie des villageois influence le mode de vie des Pygmées, soit que les Pygmées évoluent en réaction à leurs liaisons avec les villageois.

Sans entrer dans le détail, indiquons très sommairement que chaque groupe pygmée entretient des relations économiques exclusives avec des lignages ou des familles de Grands Noirs (villageois non pygmées) (Bahuchet et Guillaume 1979, Bahuchet 1984). Les chasseurs-collecteurs apportent à ces villageois une partie de leurs prises en gibier, ainsi que certains produits comme du miel ou des chenilles, et reçoivent en retour de l'outillage en fer (lames de hache, de sagaie, couteaux...), des marmites (en poterie ou en aluminium), du tabac, de l'alcool, mais aussi des produits agricoles (féculents : manioc, banane plantain). Saisonnièrement, les hommes pygmées participent au défrichage des nouveaux champs (abattage), et leurs femmes aident les femmes villageoises pour les tâches de récoltes ou de transport. Ordinairement, les deux communautés sont indépendantes en ce qui concerne leur fonctionnement social : ainsi la parenté, l'organisation sociale et la religion sont différentes. 


\section{Ce que nous apprennent les langues}

S'il est vrai que l'on ne connaît pas de "famille linguistique pygmée " (à la différence des Bushmen, dont les langues à clics appartiennent à la famille Khoi-San), la plupart des Pygmées parlent des langues individualisées, qui leur sont propres, mais qui sont apparentées avec d'autres langues africaines, des familles bantoues, oubanguiennes ou soudanaises (Thomas 1979). Cependant ces langues apparentées ne sont pas nécessairement géographiquement proches, et tous les peuples vivant de nos jours avec des Pygmées ne sont pas linguistiquement parents de ceux-ci. Par exemple les Aka de RCA vivent entourés de 19 ethnies d'agriculteurs, dont seulement 6 font partie de la même famille linguistique. Les Baka du Cameroun, quant à eux, n'ont plus aucune relation avec les locuteurs des langues oubanguiennes apparentées, qui vivent en RCA ou au Zaïre. On voit donc que beaucoup de groupes pygmées ne vivent pas de nos jours en relation avec des Grands Noirs dont les langues sont parentes (Bahuchet et Thomas 1986).

Il résulte de ces constatations que toutes les ethnies vivant aujourd'hui avec les Pygmées n'ont pas une place égale dans leur histoire ancienne, et que plusieurs groupes pygmées ont " changé de patrons " au cours du temps.

On peut schématiser ainsi cette histoire des langues (Bahuchet 1989). Les ancêtres pygmées s'associent avec des ancêtres grands noirs, en une vie commune au cours de laquelle les Pygmées empruntent la langue des Grands Noirs. Au cours d'une phase suivante, les groupes se distancient et leurs parlers évoluent séparément, jusqu'à devenir des langues différentes sans intercompréhension (les langues actuelles des Pygmées et des Grands Noirs). A la suite ou pendant cette phase de distanciation, les Pygmées créent de nouvelles alliances avec d'autres groupes de Grands Noirs, différents, avec lesquels ils n'ont pas d'histoire commune, et dont ils ne subissent pas d'influence linguistique, puisqu'ils conservent leur propre langue maternelle.

Il y a donc deux phases différentes dans l'histoire des relations entre Pygmées et Grands Noirs, ce qui complique singulièrement les questions récurrentes sur " une culture pygmée pure ", " des coutumes pygmées ", des influences culturelles...

\section{Les fondements de la relation Pygmées/Grands Noirs}

Dans la période précoloniale récente (c'est-à-dire jusqu'au début du XIX'e siècle), la relation est principalement un échange économique, association qui permet aux deux partenaires d'exploiter mieux deux écosystèmes juxtaposés et différents, la forêt et les champs. Les groupes sont complémentaires, ils divisent leurs efforts, ce qui permet de travailler moins pour obtenir qualitativement plus. Mais ce n'est pas tout.

Les biens qui résultent des échanges pénètrent au cœur des deux sociétés. Chez les Pygmées, les outils de fer entrent comme composante essentielle dans les compensations (" dot ") préliminaires aux mariages. Les mariages sont à la base de l'organisation socio-économique par la complémentarité hommefemme qui en résulte. De plus, l'alliance entre deux familles entraîne une co- 
opération entre celles-ci, marquée par des visites de longue durée conditionnant l'occupation et le partage des territoires.

Du côté des agriculteurs, on a pu montrer en République centrafricaine que la viande (gibier boucané ou fumé), obtenue auprès des Pygmées, forme les provisions abondantes nécessaires à la réunion de l'ensemble des lignages alliés, principalement lors des levées de deuil, qui marque la réactivation des alliances et la réaffirmation de l'unité de la société villageoise (Bahuchet et Thomas 1985). De même au Zaïre, ce sont les Pygmées qui approvisionnent les villageois en viande pour la tenue des grandes fêtes publiques conditionnant la sortie d'initiation des garçons.

Le système social de chaque partenaire a besoin de l'autre pour se reproduire, il est donc fondé sur l'apport de la société associée.

L'association est cimentée par des liens sacrés. La participation de l'un des groupes aux rituels de son partenaire a pour fonction principale de créer une fraternité indéfectible. Que ce soit par la participation d'un Grand Noir à l'initiation à une société d'hommes comme le Jengi des Baka au Cameroun, ou par la circoncision en commun de Pygmées et de villageois comme dans l'Ituri, les jeunes co-initiés sont frères de sang et de classe d'âge, et cette fraternité ne peut se rompre que par la mort. C'est aussi ce que signifie la participation des partenaires aux levées de deuil, ainsi lorsque le masque aka d'Ezengi se manifeste dans les deuils des villageois, leurs patrons.

L'importance mythologique des Pygmées pour les Grands Noirs est aussi une marque de l'antiquité de leurs relations. On retrouve en effet les Pygmées présents, soit nommément, soit par projection assez transparente, dans la religion, la cosmogonie et la magie. Ils apparaissent dans des rites de possession, dans les cures de thérapie traditionnelle, dans les rites d'intronisation. De très nombreuses populations forestières font intervenir des Pygmées, ou des êtres de petite taille, dans leurs récits d'origine et de peuplement, même si ces ethnies n'ont actuellement plus de relations avec des Pygmées. Les mythes de nombreuses populations de la Lobaye $^{3}$ (RCA) comme de l'Ituri (Zaïre) montrent que ceux-ci " dérobèrent " aux Pygmées des éléments aussi essentiels que le piégeage, la forge, même l'agriculture et la vie dans les villages. Les Pygmées, dépouillés, se sauvèrent alors en forêt pour $\mathrm{y}$ vivre de chasse et de cueillette. On voit le rôle ambigu que jouent les Pygmées, civilisateurs et sauveurs qui deviennent relégués et sauvages. On trouve fréquemment, justifiant l'alliance actuelle des agriculteurs avec les Pygmées, des mythes dans lesquels les Grands Noirs se définissent comme les protecteurs des Pygmées.

\section{Les forces perturbatrices}

Le schéma équilibré des relations villageois/Pygmées n'existe plus. Le cercle s'est ouvert, avec un troisième partenaire : l'Europe, qui recherchait elle aussi les produits de la forêt. On peut distinguer plusieurs grandes étapes de cette ouverture de la forêt au monde extérieur : 
- Époque précoloniale : le commerce interafricain à longue distance. L'ouverture vers l'Europe commence avant la colonisation : dès l'arrivée des Portugais au Congo au XVI ${ }^{e}$ siècle, on cherche l'ivoire, du bois rouge à teinture, puis surtout les esclaves.

- Époque coloniale : l'économie de traite. Les villageois subissent la contrainte du travail forcé, pour les compagnies concessionnaires coloniales.

- Aujourd'hui : l'envahissement. Des étrangers s'installent en grand nombre, dans des chantiers forestiers, des mines d'or ou de diamant, des plantations industrielles de café, cacao, hévéa, etc.

On verra que depuis cinq siècles, les Pygmées aident leurs patrons à répondre aux demandes du monde extérieur. C'est une longue chaîne de perturbations et d'influences...

\section{Évolution des échanges}

\section{L'époque précoloniale: le commerce interafricain à longue distance}

On sait l'importance des circuits commerciaux à Iongue distance en Afrique équatoriale, longtemps avant l'implantation des Européens. C'est d'ailleurs leur existence qui permit la mise en place du commerce de l'ivoire et des esclaves. Dès le XVII ${ }^{\mathrm{e}}$ siècle, les premiers voyageurs, tels Andrew Battel en 1625, témoignent dans la région atlantique de la participation des Pygmées comme " producteurs de base ", dans le cadre de leurs relations d'échange de type traditionnel, fondées sur le volontariat et le besoin réciproque. La description d'Olivier Dapper, publiée en 1668 (Bahuchet et Guillaume 1982), décrit déjà tout le circuit ; en même temps qu'il cite les échanges entre Pygmées-chasseurs d'éléphants et villageois-agriculteurs, il montre le rôle d'intermédiaires de ceux-ci et la finalité des échanges : les Pygmées tuent les éléphants, donnent l'ivoire à leurs maîtres qui la porte au roi du Congo pour les Européens... La place des Pygmées-fournisseurs d'ivoire a été par la suite attestée par tous les voyageurs, à travers les siècles. Logiquement, ces relations ne pouvaient au début qu'être équilibrées puisqu'elles permettaient la vie de chacune des sociétés en présence.

Toutefois le commerce des esclaves, qui se développa au XVIII siècle, fut autrement perturbateur. On sait qu'une bonne partie en était capturée dans le bassin de l'Oubangui et du Congo, ce qui provoqua la fuite des populations à l'intérieur de la forêt, et leur fit rechercher l'aide des Pygmées.

\section{L'époque coloniale: l'économie de traite}

A partir des dernières années du XIX $\mathrm{X}^{\mathrm{e}}$ siècle $^{4}$, la pénétration coloniale, en instaurant une nouvelle économie, transforma profondément le système précolonial d'alliance. Dans l'est du Zaïre, la zone forestière fut touchée dès le milieu du XIXe siècle par les trafiquants d'ivoire et d'esclaves venant du Soudan pour

4. Cf. pour l'ensemble de l'Afrique centrale, Coquery-Vidrovitch \& Moniot 1974, Suret-Canale 1977, et pour la Lobaye (RCA) Bahuchet 1985, Sévy 1972, Thomas 1963. 
atteindre le royaume mangbetu, à la lisière de la forêt (Keim 1983). Par la suite, ce fut le gouvernement égyptien qui régit cette route de commerce, mais la recherche d'ivoire persista. (Il faut souligner l'impact que produisaient ces colonnes de commerçants, groupant plusieurs centaines de personnes, sur l'économie vivrière de la région, et l'approvisionnement qu'elles nécessitaient.)

Toutes les colonies du bassin congolais (Kamerun allemand ; Afrique équatoriale française, par la suite Congo français ; État libre du Congo de Léopold II, par la suite Congo belge) subirent la mise en place de l'économie de traite, c'est-à-dire une dépendance totale à l'égard de la métropole, avec un commerce où l'on n'achetait que pour exporter et où l'on ne vendait que des produits importés. Les terres du Kamerun, du Congo français, puis du Congo belge furent réparties entre des sociétés capitalistes et groupes financiers européens (Süd-Kamerun Gesellschaft, Compagnies concessionnaires) auxquels les gouvernements assuraient le monopole d'exploitation et très souvent de commerce, sinon en droit, au moins de fait. L'impôt de capitation fut institué, mais le rapport des territoires était fixé à l'avance, et le paiement en nature obligatoirement fait aux compagnies. Le caoutchouc sauvage (" le caoutchouc sanglant ») et l'ivoire en étaient les deux principaux produits (malgré des chutes périodiques des cours), mais d'autres ressources plus locales étaient aussi recherchées à des époques diverses (bois, huile de palme, résine de copal, peaux d'antilopes, certaines noix oléagineuses).

En dehors des concessions se développèrent de vastes plantations (cacao, café, hévéa, palmier à huile...) au Cameroun comme au Congo et au Congo belge, où s'ouvrirent aussi d'importantes mines. Ces entreprises nécessitèrent une nombreuse main-d'œuvre et l'établissement du travail forcé. De même, l'installation des Européens provoqua l'ouverture de nombreux chantiers publics et mobilisa les forces pour le portage, l'ouverture et l'entretien des pistes et enfin la création des lignes de chemin de fer. Dans les années 20, les chantiers absorbaient au Moyen-Congo $40 \%$ de la population mâle âgée de 20 à 40 ans. Il y eut à ce moment-là des réquisitions continuelles et les déplorables conditions de travail en firent de réelles déportations. La mortalité était considérable et rares furent ceux qui en revinrent. Toutes les régions furent touchées, d'abord les plus proches des chantiers et, rapidement, les plus éloignées (MoyenCongo, Gabon, Oubangui et même Tchad...). Le seul chemin de fer CongoOcéan (1921-1934) tua plus de 20000 hommes et, des chemins de fer congolais, on disait « un mort par traverse ». Le dépeuplement rural en forces vives fut très important et la déstructuration profonde.

A la période d'exploitation, ou mieux « d'extrativisme ", succéda celle des cultures obligatoires. Dans de nombreux territoires, on imposa aux paysans la culture de plantes commerciales (café, hévéa, cacao et même coton) dont l'achat des produits était le monopole des compagnies concessionnaires.

Dans tous les cas, la monoculture d'exportation était possible grâce à l'impôt et au travail forcé, et corollairement elle entra dangereusement en compétition avec les cultures vivrières. Un grave état de déficience nutritionnelle s'ensuivit.

L'éclatement des deux guerres mondiales eut des conséquences désastreuses pour les colonies lorsque les métropoles exigèrent des " efforts de guerre ", tout à la fois en recrutant des soldats (200 000 hommes en AEF/AOF pour 
la guerre 14-18) et en intensifiant la production de produits stratégiques (caoutchouc et vivres) destinés à l'exportation. Le caoutchouc fut repris en 1939 après un abandon de vingt ans. Tout cela accéléra les déplacements de population et désorganisa les rythmes vivriers, générant famines et révoltes.

Les populations forestières n'échappèrent évidemment pas à ce mouvement général. Certaines régions furent extrêmement touchées (Moyen-Congo, Oubangui), d'autres moins durement (Cameroun) mais les conséquences économiques furent partout sensibles. Par contrecoup, les groupes pygmées qui n'avaient jamais été victimes directement du régime colonial (ni réquisition, ni travail forcé), du fait de leur nomadisme, subirent les pressions de leurs alliés villageois pour les aider à répondre aux besoins des colons : intensification de la chasse à l'éléphant pour l'ivoire (l'AEF en exporta plus de 100 tonnes par an de 1896 à 1900, pour monter jusqu'à 160 tonnes par an en 1910), intensification de la chasse d'approvisionnement quand le travail forcé rendait les villageois incapables d'assurer leur subsistance, production de viande aussi pour les postes coloniaux et production de peaux d'antilopes pour le cuir au lendemain de la guerre de 14-18 (plusieurs centaines de milliers par an jusque dans les années 50 - ainsi, par exemple, 800000 peaux exportées en 1937 pour l'AEF). Au Congo belge, on cherchait l'ivoire mais aussi le cuir de buffle de forêt.

A certains endroits, l'administration coloniale tenta de sédentariser les Pygmées (ce qu'on a appelé "politique d'apprivoisement ") et de les adapter à l'agriculture (dès 1933 dans le nord du Moyen-Congo par exemple).

Le système ancien d'alliance fondé sur le besoin réciproque se transforma en conséquence en un système plus autoritaire, la brutalité coloniale provoquant par résonance un durcissement des relations entre les patrons Grands Noirs et leurs Pygmées.

L'économie de marché qui suivit l'économie de traite, dans les dernières années de colonisation et après les indépendances, maintint ce type de relations où les villageois tendaient à considérer les Pygmées comme une main-d'œuvre servile à leur libre et entière disposition.

Ce fut aussi à partir du moment où les villageois durent cultiver les plantes de rente - ce qui entra en compétition avec l'agriculture vivrière - , que ceux-ci utilisèrent leurs clients pygmées comme main-d'œuvre servile et nécessaire, soit dans les champs soit dans les plantations. C'était vital pour qu'ils survivent dans ce système colonial, et cela marqua une étape cruciale dans leurs relations avec les Pygmées.

Si les indépendances mirent fin aux exactions directes, il nous faut encore rapporter quelques circonstances particulières où les Pygmées eurent un rôle à jouer. Dans certaines régions, les années suivant l'indépendance (1960-1970) furent marquées par des mouvements de rébellion contre le pouvoir central, guérillas qui provoquèrent l'envoi de troupes militaires pour sillonner les forêts (mouvements de l'UPC au sud-est du Cameroun ; révolte des Simba dans la région de l'Ituri au Zaïre). A plusieurs reprises, des groupes de Pygmées s'y trouvèrent mêlés, utilisés soit par les militaires nationalistes, soit par les rebelles, comme guides et pisteurs. 


\section{SITUATION CONTEMPORAINE}

\section{La situation économique}

Si la période coloniale est marquée par l'économie de traite et le travail forcé, la période contemporaine est caractérisée par l'envahissement de la forêt. C'est la forêt elle-même et son sous-sol qui sont convoités, attirant des populations étrangères à la région, qui s'y installent pour y travailler : exploitation du bois, plantations industrielles de rente (café, cacao, hévéa, palmier), ainsi que mines d'or et de diamant.

Les grandes tendances du développement économique des régions forestières influencent directement le style de vie des habitants de la forêt. Plutôt que de passer en revue les diverses régions, il est plus judicieux de dégager une typologie des cas contemporains, qui sont tous observables dans tous les pays habités par des groupes pygmées.

En premier lieu vient évidemment l'exploitation du bois qui a pris un développement considérable ces quinze dernières années. Les quelques entreprises artisanales disséminées des années précédant l'indépendance ont fait place à de puissantes sociétés étrangères ou mixtes, aux moyens importants, travaillant sur des concessions immenses. A titre d'exemple, le volume de fûts abattus en RCA a quintuplé en vingt ans, et il est quarante fois plus important qu'il y a quarante ans (12000 mètres cubes en 1946, 90000 en 1960 et 480000 en 1980). C'est en RCA que les surfaces exploitées sont les plus vastes par rapport à la superficie d'ensemble (1500 000 hectares soit $50 \%$ de la totalité) ; au Cameroun et au Congo, la superficie sous licence d'exploitation est de l'ordre de $25 \%$ de la surface forestière totale.

L'exploitation forestière agit à deux niveaux sur une région.

- L'impact écologique est évident, bien que paradoxalement " indirect " c'est plus par la lourdeur de l'infrastructure que par l'abattage proprement dit que l'exploitation perturbe profondément l'écosystème en morcellant la forêt (ouverture des routes d'accès et aires de débardage).

- L'impact socio-économique est non moins important. Les chantiers d'exploitation, généralement doublés d'une scierie, provoquent la création d'agglomérations d'ouvriers, avec une majorité d'étrangers à la région exploitée, elle-même généralement sous-peuplée. De plus, ces nouveaux villages sont d'ordinaire établis dans des lieux auparavant inhabités ou à proximité de tout petits hameaux. Cet afflux de population entraîne une modification profonde des réseaux d'approvisionnement.

Il en va de même lors de l'ouverture de plantations industrielles sur de vastes surfaces (café, cacao, hévéa, palmiers à huile). L'impact écologique, bien que différent de celui de l'exploitation forestière, peut être considérable si les surfaces sont très vastes (comme Hévécam au sud-ouest du Cameroun : 35000 ha).

Les implantations minières d'or et de diamant (Cameroun, RCA, Zaïre) provoquent également un accroissement conséquent de la population, lequel entraîne fréquemment un état d'insécurité, voire d'anarchie très grave. 
Le développement économique régional provoque un accroissement des villes (préfectures, sous-préfectures), entraînant à la fois une augmentation du marché, et un élargissement des surfaces déboisées autour de l'agglomération.

En résumé, les conséquences du développement des régions forestières se marquent par une diminution générale des surfaces boisées, un morcellement de la forêt, un accroissement sensible de la population, souvent exogène, et une augmentation de la demande en vivres.

Les réactions des groupes pygmées à une telle situation sont assez diverses, dans les régions que nous connaissons. On rencontre en effet partout un continuum, depuis les campements conservant une économie d'échange de type traditionnel, jusqu'aux employés salariés.

\section{Situation traditionnelle}

Les liens héréditaires avec des familles de villageois sont maintenus. Les Pygmées, restant mobiles, échanges préférentiellement avec ces agriculteurs, leur fournissant viande fumée et produits de cueillette. Ils reçoivent en retour de l'outillage, des vivres (plantes cultivées), du tabac, de l'alcool, des habits et, rarement, de l'argent. Ils viennent périodiquement travailler dans les champs de " leurs " villageois, principalement lors des défrichements, ainsi que lors de la récolte du café ou du cacao. La principale différence avec un "vrai " système « traditionnel » réside dans la commercialisation importante de viande que réalisent les « patrons » des Pygmées. On a vu précédemment qu’une telle situation s'était installée dès la colonisation, alors qu'aux époques précoloniales, seul le surplus de viande provenant des chasses pygmées allait au village, où il était consommé ; les chasseurs sont désormais les premiers producteurs de viande pour alimenter, par personne interposée (le patron villageois), un commerce extérieur à l'ethnie.

De nombreux campements de RCA (basse Lobaye, Sangha) et du Zaïre (nord de l'Ituri) vivent de cette manière.

\section{La production de viande}

L'extension de ce système passe par la disparition de l'intermédiaire héréditaire ancestral (par exemple, lorsqu'à la suite de conflits un groupe local migre). Il est maintenant fréquent que des acheteurs de viande, étrangers à la région, se rendent directement sur le lieu de production, dans les camps de chasse en forêt, soit avec des vivres, soit avec de l'alcool et de l'argent, et achètent sur place le gibier qu'ils iront revendre dans les villages d'entreprises (par exemple, région de Loko en RCA).

A l'extrême limite, on assiste à un réel parasitage des campements par les acheteurs de viande qui viennent effrontément s'installer et restent sur place jusqu'à ce que leur budget soit épuisé (région de Ndèlè en RCA, sud de l'Ituri au Zaïre). On peut ainsi trouver dans un campement deux ou trois acheteurs, résidant là plusieurs semaines de suite sans que les Pygmées puissent ou osent les chasser. 
Dans le nord de l'Ituri, ce sont les chasseurs pygmées qui font de temps en temps des expéditions de commerce vers de petites villes, échangeant leur viande contre des biens (tissus, machettes, huile de palme). En effet les villages d'entreprises forment des pôles d'attraction, des marchés libres d'accès auxquels les Pygmées peuvent se rendre sans l'intermédiaire de leurs associés-patrons.

La spécialisation dans la "production » de viande avec vente « directe » s'accompagne fréquemment d'une location de la force de travail. Les Pygmées, ayant rompu les relations héréditaires, se trouvent ainsi libres de travailler périodiquement pour le plus offrant, soit au défrichage des champs, soit plus souvent à la récolte des cultures de rente. Les sommes d'argent obtenues sont cependant généralement dérisoires (3 à 4 francs français par journée de travail). C'est le cas, par exemple, des Bongo de la région de Sibiti (Congo).

\section{La ruralisation in situ}

Le passage à l'agriculture chez les Pygmées est dorénavant un mouvement important. Balbutiant dans certaines régions (Lobaye, Ituri), il est ancien dans d'autres (Nord-Congo - région d'Enyèlè - côte du Cameroun) et moins ancien mais néanmoins général ailleurs (est du Cameroun). On trouve là encore de nombreux degrés, depuis le petit jardin d'appoint jusqu'à la vaste bananeraie qui autorise la commercialisation d'un excédent de production ; à côté de l'agriculture l'activité de chasse, variable selon les groupes, se poursuit : certains font alterner de longues saisons de chasse en campements de forêt avec une saison agricole au village « de base ", alors que d'autres résident en permanence au village, ne sortant en forêt que pour quelques semaines par-ci par-là. Il n'est pas rare que ces mêmes Pygmées aient également créé une plantation de rente (café ou cacao). Rien ne les distingue plus alors, économiquement, de nombre de villageois non pygmées des mêmes régions. Cependant la situation de ces Pygmées ruraux ne laisse pas d'être complexe car beaucoup continuent aussi d'entretenir les relations de type héréditaire avec les villageois Grands Noirs, tant d'échange que de travail temporaire sous la forme de corvées.

Dans les villages twa (région du lac Tumba, Zaïre), situés le long de la route principale, toutes les familles exploitent des champs de manioc (dont ils vendent une partie de la production), en quantité moindre cependant que leurs voisins oto (moins de 2 champs par famille chez les Twa, contre près de 3 chez les Oto). Par contre, à moins de 30 kilomètres de là sur des pistes secondaires non fréquentées, les familles pygmées possédant des champs ne forment plus que de 65 à $85 \%$ de l'ensemble (selon les villages) et le nombre de champs est encore plus faible (pas plus d'un champ par famille, contre 2,5 chez les Oto), en même temps que la surface en est très réduite (en moyenne moins de 2000 mètres carrés, contre $1 / 2$ ha chez les villageois oto). Dans cette région toutefois, en 1990, la récession économique entraîne une baisse considérable du pouvoir d'achat de la population, ce qui n'assure plus aux Twa, nouveaux paysans, le minimum vital. Les villages sont actuellement peuplés d'une majorité de Twa improductifs, qui ont peine à trouver leur nourriture.

Les Kola du sud-ouest du Cameroun forment un bon exemple de cette ruralisation d'un type particulier. Les familles, installées dans des villages perma- 
nents à l'intérieur des massifs forestiers, entretiennent des champs vivriers généralement de surface médiocre (moins d'un demi-hectare), et qui souvent sont insuffisants pour l'approvisionnement. L'activité principale reste néanmoins la chasse, effectuée à proximité du village une partie de l'année, mais aussi dans des camps de chasse assez éloignés, où l'on reste pendant plusieurs mois. Pendant ces séjours en forêt, les hómmes recherchent également pour les revendre plus tard les graines de Strophantus à usage pharmaceutique. La viande est ensuite vendue ou échangée contre alcool, vivres et tabac, souvent sur les lieux mêmes du campement, où les revendeurs viennent pour des séjours quelquefois durables.

Certains Bongo de la région de Sibiti (Congo) ne pratiquent plus qu'une chasse réduite mais approvisionnent le marché local en bois de chauffage et... en volaille !

\section{Ruralisation et émigration}

A l'initiative des pouvoirs publics ou de sociétés religieuses de bienfaisance sont quelquefois créés des villages-pilotes spécialement destinés aux Pygmées. L'implantation de ces villages est souvent choisie dans des zones étrangères aux Pygmées que l'on y déplace (ou qui choisissent de s'y installer), en fonction de potentialités agricoles ou d'opportunités socio-économiques. Une rupture spatiale aidera, pense-t-on, à une nécessaire rupture sociale des relations avec les villageois agriculteurs. Des animateurs ruraux partagent la vie du village et cherchent à former les nouveaux paysans à une agriculture efficace. Toutefois les activités de chasse restent présentes, même si elles deviennent limitées dans le temps.

\section{Les travailleurs saisonniers}

Il s'agit là d'une mutation économique qu'effectuent des groupes locaux ayant ou non des rudiments d'agriculture (le plus souvent sans). Ceux-ci rompent leur relation avec les villageois et viennent s'engager comme saisonniers dans de grandes plantations industrielles de café, au moment de la récolte. Là, pendant les trois mois de la récolte et de l'usinage, ils sont salariés et nourris par le planteur. La saison terminée, ils repartent en forêt mener leur vie de chasse et de producteurs de viande sauvage, ce qui leur assure, après échange, l'approvisionnement en féculents cultivés. C'est le cas en Lobaye, vers Boda.

Certains Efè (nord-est de l'Ituri) travaillent ainsi pour de " petits planteurs » de café, pendant les quelques semaines que dure la récolte ; ils sont payés, souvent en nature, à la fin de la saison, et nourris pendant leur séjour à la plantation. Ils trouvent aussi le temps de chasser.

Dans la palmeraie de Mokeko (Sangha, Congo), 90 Pygmées sur 1100 ouvriers sont employés au débroussaillage et au défrichement des parcelles. Considérés comme permanents pour la plupart d'entre eux, ils montrent cependant un très fort absentéisme et une grande irrégularité, ne travaillant pas plus de 
quinze jours sur les 25 ouvrables du mois. La chasse au filet et au fusil est largement pratiquée. Très monétarisés, ils emploient l'argent dans leurs propres tractactions.

\section{Le salariat}

Certaines sociétés forestières emploient des Pygmées, à des titres divers. Fréquemment manœuvres (pour la manutention par exemple), ils peuvent aussi être ouvriers aux machines, voire sur les chantiers d'abattage. Il leur arrive même d'être manœuvres pour ouvrir les layons dans les camps de prospection.

Ainsi à Bayanga (usine Slovenia-Bois) en 1979, de nombreux Pygmées étaient manœuvres pour les camps de prospecteurs. L'ensemble de la communauté se déplaçait sur les lieux et y installait un campement pygmée, à côté du camp des prospecteurs. Ils restaient là plusieurs mois avec leur famille entière, avec des retours périodiques à Bayanga (une fois par an), véhiculés en camionbenne, pour les paies et les marchés. Durant leur séjour, les hommes travaillaient à leur tâche, utilisant leur temps libre (l'après-midi) à chasser ou piéger, pendant que les femmes cueillaient. De cette manière, ils approvisionnaient aussi les autres employés.

C'est aussi le cas à Ouesso (Congo) où l'exploitation forestière de Pokola emploie une cinquantaine de Pygmées (20\% de l'effectif), mais seulement 9 d'entre eux comptent plusieurs années d'ancienneté. Employés à la prospection ou à l'abattage (comme « aides scieurs », c'est-à-dire porteurs de bidons d'essence), ils sont déplacés en famille sur les lieux de chantier ; à l'entreprise, ils préfèrent vivre en camp à côté du village. Ils continuent à chasser, mais, salariés, achètent de la viande... aux Pygmées ! Ils achètent aussi les produits agricoles et l'alcool.

A la Siplac (sur la Mbaéré, RCA), de nombreux hommes sont employés à l'usine comme ouvriers. Ils vivent avec leur famille dans un des " quartiers » du village d'entreprise, dans des maisons de style villageois, en terre. Les temps libres sont employés à la chasse aux filets, alors que les femmes entretiennent des champs vivriers. Périodiquement, des membres de leur parenté non employés viennent leur rendre visite, installant leur camp de huttes près des maisons, chassant de concert et échangeant des produits féculents ; les Pygmées salariés jouent ainsi le rôle de villageois agriculteurs.

De nombreux Twa du lac Tumba sont employés en permanence ou temporairement, souvent en bas de l'échelle des salaires, dans les plantations industrielles d'État ou privées qui bordent le lac (hévéa, café, cacao). Dans le meilleur des cas, les employés permanents bénéficient d'une certaine assistance sanitaire et d'un « économat » (magasin de l'entreprise vendant à meilleur compte). Les Twa salariés n'ont que rarement des champs ; ils achètent au marché leur nourriture. Une particularité leur revient, celle d'élever des volailles qu'ils vendent ou échangent aux autres villageois. Les visites de Twa non employés sont fréquentes et peuvent être durables. A ces occasions, les parents venant de la zone forestière apportent avec eux de la viande de leurs chasses et contribuent ainsi à faire accepter leur séjour. 


\section{Les citadins}

Dans de très rares cas, quelques Pygmées migrent vers des villes ou bourgades de province et y vivent pauvrement dans les faubourgs, subsistant de basses besognes, sombrant dans l'alcoolisme et la prostitution (Mambasa au Zaïre par exemple).

Qu'ils soient producteurs de viande de gibier, agriculteurs ou employés, dans tous les cas les Pygmées contemporains accordent encore une grande importance au milieu forestier, à la chasse et au piégeage, comme à la collecte qui vient toujours compléter les produits agricoles. Un autre domaine, où les Pygmées conservent une grande notoriété, est celui de la médecine traditionnelle et de la magie. Les devins-guérisseurs sont fréquemment consultés et nombre d'entre eux sont célèbres régionalement.

Deux points méritent d'être soulignés, que nous approfondirons par la suite. En premier lieu, il faut insister sur le fait que la sédentarisation n'entraîne pas nécessairement la rupture des relations ancestrales avec les villageois, et surtout qu'elle ne supprime pas les rapports d'exploitation. Au contraire, dans certains cas, elle les facilite. En second lieu, les Pygmées montrent souvent une préférence pour des travaux journaliers à rendement immédiat, plutôt que pour une agriculture suffisante dont le rendement est différé, même là où les groupes ont déjà établi des champs.

\section{Les problèmes sociaux}

Si dans la majorité des cas les groupes pygmées réussissent une insertion économique qui assure leur subsistance, les problèmes sociaux ne manquent pas et sont souvent graves. Ils se manifestent à plusieurs niveaux, dans les relations internes à la société, dans les relations avec les autres habitants des régions et, en dernier lieu, dans les rapports avec l'administration.

\section{Les conditions de vie}

En dehors des rares campements entièrement forestiers et des villages permanents de certains groupes devenus agriculteurs, l'habitat de la plupart des Pygmées laisse perplexe. En effet, la tendance générale a été une diminution sensible du rythme migratoire (on est passé de 6 fois par an à 2 ou 3 ) ; des campements faits pour durer deux mois ont laissé place à des «villages » semipermanents où l'on vit plus longtemps d'affilée mais toujours dans des huttes ou de méchantes cabanes de palmes, rarement solides. L'élimination des déchets, simple en cas de semi-nomadisme, devient problématique lorsque l'on se fixe, de même que l'entretien des abords des points d'eau et, de ce fait, l'hygiène est précaire. L'habitat éphémère s'adapte mal à une vie durable.

Ajoutons que cette sédentarisation naissante s'accompagne souvent d'un regroupement et de l'augmentation sensible de la taille des groupes (on passe, par exemple, de 70 personnes à plusieurs centaines, voire un millier). 


\section{La vie sociale}

Traditionnellement acéphales, les ethnies pygmées se sont vues partout obligées d'accepter la tutelle administrative du chef de village (soit pygmée, soit villageois), en même temps que l'évolution des rapports avec les villageois entraînait la nécessité d'un "chef ", c'est-à-dire d'un interlocuteur responsable celui à qui l'on donne les ordres. Polarisation des groupes donc, mais qui n'est pas toujours admise par l'ensemble des Pygmées. Dans un même temps, la sédentarisation favorisant l'ouverture des jeunes sur le monde moderne provoque des conflits fréquents entre générations, lorsque les jeunes refusent les conseils de leurs aînés. La fixation de villages le long des routes et la limitation des déplacements entraînent aussi une oisiveté assez marquée chez les jeunes garçons qui sont moins attirés par la chasse que leurs parents. Néanmoins, cet état ne saurait durer au-delà du mariage qui les oblige alors soit à prendre en charge une plantation vivrière, soit à chasser un minimum.

La société traditionnelle (Aka, Baka) accordait une influence prépondérante à trois personnages : l'aîné du campement, le maître de la chasse à l'éléphant et le devin-guérisseur, chacun ayant sa propre sphère de compétence. Le monde moderne ne voit plus guère opérer que le devin-guérisseur, dont le rôle a pris de l'importance, jusqu'à l'amener à intervenir dans des rituels de chasse auparavant hors de sa compétence.

La structure socio-économique elle-même est évidemment fortement ébranlée par les nouvelles conditions de subsistance. Là où prévalait une appropriation collective des ressources s'installe une individualisation des produits, qui gauchit les systèmes de partage et de circulation des biens. La valeur fiduciaire de la viande destinée à la vente rend caduc le système de partage du gibier, le chasseur préférant conserver entière sa prise pour l'échanger ou la vendre au village.

\section{Santé}

Bien que la sédentarisation influence défavorablement l'hygiène, il est difficile de déterminer si l'état de santé des Pygmées s'est modifié, dans la mesure où les conditions de vie traditionnelles étaient déjà physiquement éprouvantes. On note cependant une augmentation de la charge parasitaire (en relation avec la promiscuité) ce qui influe sur les anémies. On observe aussi que la maladresse dans la construction des maisons rectangulaires mal closes, liée au dénuement vestimentaire, rend les nouveaux villageois plus sensibles au froid de changement de saisons que dans les huttes traditionnelles, et de ce fait, plus sujets aux affections broncho-pulmonaires. La sédentarité des villages entraîne aussi le pullulement des puces-chiques qui prend ainsi allure de fléau et provoque des ravages sur les pieds et même les mains des jeunes enfants.

Pour quelques points particuliers, les dégradations sont sensibles. En premier lieu, chez les Pygmées comme de nombreux peuples ruraux du tiers-monde, l'ivrognerie est très importante et sans frein. Outre le fait que l'alcool et le chanvre entrent fréquemment dans les monnaies d'échange des villageois, les Pygmées utilisent souvent l'argent qu'ils ont pour en acheter, en s'endettant 
éventuellement à l'avance auprès des revendeurs qui leur extorquent ainsi la quantité de viande qu'ils veulent.

En second lieu, l'équilibre nutritionnel se trouve souvent compromis, en dehors des groupes où la chasse est encore très importante. Parmi les groupes plus sédentarisés, agriculteurs ou salariés, la mobilité réduite et l'indisponibilité rendent les actions de chasse moins fréquentes et moins efficaces, et donc restreignent l'approvisionnement. La régression générale du gibier dans de nombreuses régions, à cause de l'augmentation de la population sédentaire, des exploitations modernes et du commerce de viande, contribue aussi à cet appauvrissement du régime alimentaire.

Dans d'autres régions (comme celle du lac Tumba), les hommes adultes, attirés par les emplois dans les entreprises, abandonnent dans les villages de forêt, les vieillards, les femmes et les enfants, ainsi dépourvus d'approvisionnement carné régulier. Dans ces conditions, les catégories les plus vulnérables (enfants, vieillards) sont nettement défavorisées et souffrent de malnutrition. La disparition des systèmes de partage entraîne d'ailleurs souvent le délaissement des vieillards et un désintérêt pour leur sort de la part des plus jeunes qui ne se préoccupent plus de les nourrir (Twa, Kola). Dans ces mêmes régions, le passage exclusif à une activité mal maîtrisée (l'agriculture) entraîne des catastrophes nutritionnelles car les paysans débutants sont incapables de pourvoir à leur approvisionnement, tout en étant coupés d'autres sources (échanges avec les villageois rompus, mais refus du retour à la forêt).

En dernier lieu, il apparaît, au moins dans certaines régions où la documentation existe, des variations dans la démographie, accusant une légère baisse, ainsi qu'un déséquilibre en faveur des villageois, ce qui est un indice net de conditions sanitaires défavorables pour les Pygmées. Ainsi, près du lac Tumba, le nombre d'enfants (nés vivants) par femme chez les villageois est passé en dix ans de 4,05 (1960) à 5,70 (1970), alors qu'il ne variait guère chez les Twa (de 4,94 en 1960 à 4,75 en 1970).

Ailleurs, sans que des chiffres soient disponibles, les premières observations montrent aussi un net déficit dans les naissances (Kola du sud-ouest du Cameroun) - c'est-à-dire que dans de nombreux villages les enfants sont en minorité ( $42 \%$ de la population) - alors que les enfants sont nombreux chez les Baka et les Aka (où les moins de 20 ans représentent plus de $50 \%$ ).

\section{L'argent}

C'est peut-être dans le rapport des Pygmées avec l'argent que se manifestent le plus nettement les problèmes qu'ils éprouvent à s'adapter au monde moderne. Société non monétarisée où la circulation des biens était très large, les groupes pygmées se voient confrontés au problème de la numération et du calcul de rentabilité, sans avoir reçu les moyens de les comprendre. Actuellement, continuellement trompés sur la valeur des produits qu'ils échangent, sans posséder la maîtrise des lois du marché, il leur est très difficile de rester " égalitaires " dans leur propre système social, et d'évoluer avec assurance dans le monde de l'argent. Tous les employeurs occupant des Pygmées, d'un bout à l'autre de la forêt, remarquent pour s'en plaindre et s'en étonner, l'incroyable 
inconstance de leurs employés, qui souvent disparaissent un beau jour, sans même attendre le salaire de fin de mois. On constate ailleurs qu'il est fréquent de ne travailler que le délai nécessaire pour avoir l'argent dont on a besoin pour un achat précis ou encore, de prendre à l'économat de l'entreprise, à crédit (« bon pour »), un produit quelconque que l'on revend aussitôt, moins cher, uniquement pour avoir de l'argent liquide afin de s'acheter un aliment convoité.

Ailleurs les Pygmées acceptent des salaires misérables, largement inférieurs aux taux minimaux légaux, comme ils se contentent souvent d'une contrepartie en nature ; là aussi, il s'agit souvent d'alcool ou de chanvre.

Ailleurs encore (sud-ouest du Cameroun), les succès cynégétiques d'un chasseur ou la renommée d'un guérisseur leur assurent des revenus monétaires considérables, cependant que l'on observe une grande maladresse dans l'usage de cet argent. Les biens mobiliers, les ustensiles, l'habillement sont fréquemment défectueux, alors même que l'argent est utilisé pour acheter tabac, vin ou alcool distillé localement, consommés en groupe.

\section{L'école}

La situation scolaire des Pygmées est, elle aussi, un excellent révélateur des problèmes d'intégration. En effet, même dans des régions où l'ensemble de la population est bien scolarisée (comme au sud du Cameroun), les Pygmées sont partout massivement analphabètes et très peu d'entre eux fréquentent l'école (de l'ordre de 2 à $5 \%$ au Cameroun par exemple). Chaque pays connaît le cas « du Pygmée qui est allé au lycée » et qui, soit a interrompu sa formation pour s'enfuir en forêt, soit, l'ayant achevée, se trouve rejeté par les siens comme par les villageois et sombre dans l'ivrognerie et la marginalité. Sans insister sur les régions où il n'y a pas ou peu d'écoles (RCA), celles où les villageois n'admettent pas les Pygmées dans les classes (sud-ouest du Cameroun), nous examinerons les cas où les écoles existent (est du Cameroun, lac Tumba au Zaïre).

L'obstacle principal est lié à la mobilité des familles pygmées, qui entraîne les enfants en forêt pour les saisons de chasse ou de collecte, et de ce fait les retire de l'école pour plusieurs mois de suite. Le second obstacle est celui de la promiscuité avec les villageois qui intimident fortement les enfants pygmées, provoquant l'abandon de la classe à cause de moqueries répétées. Ce peut être le cas dans des arrondissements comme celui de Moloundou (sud-est du Cameroun) où les enfants pygmées ne représentent que $13 \%$ des élèves (373 Pygmées pour 2800 villageois). En dernier lieu, un découragement est perceptible, mais il n'est pas propre aux seuls Pygmées, une sorte de " à quoi bon ? " devant une scolarisation dans des zones reculées et délaissées, où en fait l'instruction est inutile car inemployée. Il faut toutefois souligner un effet très pernicieux de la scolarisation, observable chez les Twa du lac Tumba. L'éducation reçue à travers l'enseignement valorise l'agriculture, sans toutefois en donner les moyens. Aussi les populations twa aspirent-elles à devenir agriculteurs, pour cesser d'être "des sauvages » (selon leur propre expression), ce qui les conduit à refuser de s'approvisionner en forêt en nomadisant, mais les entraîne du même coup vers une sévère pénurie alimentaire. 
La scolarisation mal conçue a un effet très grave par le fait que l'école coupe les enfants de l'apprentissage ethnoécologique que leur fournissent d'ordinaire les adultes, et qu'en valorisant d'autres styles de vie elle introduit le doute ou la honte dans l'esprit des enfants, accentuant les querelles de générations.

\section{Pygmées et villageois}

Les rapports entre ces deux populations deviennent souvent conflictuels, en relation avec l'affaiblissement des relations traditionnelles (afflux d'étrangers à la région) et avec le détournement du système par les villageois (vente et profit recherché). Cela se traduit par une hostilité jalouse des anciens " propriétaires " qui voient leurs clients se détourner d'eux pour travailler chez d'autres, et également par une tendance abusive à sous-payer la main-d'œuvre pygmée en jouant sur l'ignorance et l'attrait pour l'alcool. Néanmoins, les comportements sont souvent ambigus car les villageois sont heureux de voir " leurs » Pygmées se mettre à cultiver et, de ce fait, inconscients de ce que cela présage de séparation (puisqu'une des raisons des attaches est l'approvisionnement en vivres agricoles) ; mais, en même temps, ils apprécient assez peu la présence de ces mêmes Pygmées dans les écoles ou les dispensaires. Par ailleurs, si l'on rencontre des hommes villageois qui épousent des femmes pygmées, c'est osons le dire, faute de mieux (la dot est moins élevée), et on ne rencontre jamais de femme villageoise épousant un homme pygmée. La barrière raciale est assez nette, et les villageois manifestent toujours une supériorité sociale sur les Pygmées, même quand ceux-ci se révèlent meilleurs agriculteurs (cela existe, comme dans l'est du Cameroun), voire plus riches planteurs de cacao (on signale même des vols de récolte pygmée par les villageois !).

Le passage à l'agriculture peut d'ailleurs poser des problèmes d'ordre foncier, qui se conjuguent avec une volonté de distance spatiale et qui conduisent les Pygmées dans de nombreux cas, à s'installer très loin des villages des Grands Noirs (de deux heures à une journée de marche, pour les Kola et les Aka).

\section{Les relations avec l'administration}

En droit, dans aucune des républiques de l'Afrique équatoriale, les Pygmées ne se distinguent des autres groupes ethniques. Par exemple, la constitution de la République centrafricaine indique en préambule : " Il n'a y dans la RCA ni sujet, ni privilège de lieu de naissance, de personne ou de famille. » Un décret de 1966 insiste encore sur l'égalité de tous les citoyens en interdisant " toute mention dans les actes officiels ou sous seing privé, imprimés, formulaires administratifs ou privés, de race, de tribu ou d'ethnie ».

Le Zaïre rappela dans les années 70 cette égalité, en favorisant une campagne officielle de « réhabilitation » des Pygmées et en interdisant la discrimination (en supprimant par exemple dans le recensement la différence entre Pygmées et non-Pygmées). De ce fait, on ne trouvera dans aucun pays de texte officiel discutant du problème des Pygmées, la position extrême étant même de nier leur existence car " il n'y a que des citoyens ». Cependant la république populaire du Congo dans son dernier recensement distingue, d'une manière 
involontairement savoureuse, les "Pygmées » des "non-Pygmées "... Néanmoins il n'y a nulle part de statut juridique spécial pour les Pygmées.

Toutefois, en dépit de cette égalité officielle, les relations des Pygmées avec l'administration sont assez difficiles, comme pour toute population semi-nomade et analphabète. L'obstacle principal réside dans l'accès à l'état civil. Si rien officiellement ne les en écarte, tout en fait y contribue. Pour obtenir une carte d'identité, il faut partout un acte de naissance dont la majorité des Pygmées sont dépourvus, ce qui constitue un obstacle à peu près insurmontable lorsque l'officier d'état civil responsable de l'arrondissement n'est pas conciliant. L'absence de papiers d'identité entraîne des conséquences multiples, telles l'impossibilité de se déplacer selon les moyens normaux de circulation (cars, accès aux villes) ou encore l'impossibilité de faire appel à la justice en cas de vol ou de brutalité, et les place en butte à toutes les chicanes des forces de l'ordre. La volonté politique d'intégration pourrait se mesurer au degré de facilité d'obtention des pièces d'identité.

Second critère de la citoyenneté, la soumission à l'impôt. C'est une préoccupation constante des autorités (dès avant les indépendances, les administrations se demandaient comment assujettir les Pygmées à l'impôt), à laquelle fait effectivement obstacle la mobilité des semi-nomades. La volonté marquée de les sédentariser n'est certainement pas innocente !

La tentation est grande cependant pour des autorités locales d'utiliser la main-d'œuvre servile fournie par les Pygmées, pour toutes sortes de corvées mal rémunérées, allant de la danse lors de fêtes officielles, au désherbage des routes et abords de bâtiments publics, en passant par les réquisitions de viande par la police (dans l'Ituri), le travail dans le champ du chef de village (Ituri encore), voire la chasse au gros gibier pour des notabilités citadines. Notons aussi, comme un cas particulier, la création au Zaïre d'une compagnie de Pygmées dans l'armée, en prévision d'actions en région forestière.

Si les gouvernements n'accordent pas un statut particulier aux Pygmées, quelques-uns se préoccupent de leur sort et reconnaissent que les Pygmées font problème. Au fil des années un certain nombre d'actions ont été engagées, avec des succès divers. Ainsi au Cameroun, diverses ordonnances préfectorales provoquèrent dans les années 60 un regroupement de Pygmées le long des routes. Cependant ce mouvement ne fut pas accompagné de moyens financiers nécessaires à une action sociale, aussi les Pygmées délaissés, ayant été mal reçus par les villageois jaloux de leurs terres, refluèrent vers la forêt une dizaine d'années plus tard. On note actuellement dans le même pays une résurgence de cette politique.

Dans l'est du Zaïre, les autorités régionales cherchèrent à obliger les Pygmées à cultiver des champs en punissant d'amendes ou de prison les récalcitrants. Le résultat fut un simulacre d'agriculture (ouverture de petites parcelles par la suite délaissées).

Ailleurs (RCA), l'action auprès des Pygmées est laissée à l'initiative privée (organisations religieuses). 


\section{Les politiques de développement}

Toutes ces actions ou tous ces projets, qu'ils soient appliqués ou non, sont régis par les même principes théoriques qui se résument à trois mots : sédentarité, émancipation et agriculture.

On considère en effet que la sédentarisation est un préliminaire nécessaire à tout programme de développement (on dit aussi « d'intégration »), de même qu'une rupture des relations avec les villageois apparaît obligatoire, dans la mesure où elles sont considérées comme humiliantes, étant généralement vues comme une forme d'esclavage désormais périmée. On prône en même temps le développement d'une agriculture pygmée, considérée comme facteur à la fois de libération de l'emprise des villageois et d'intégration à la vie nationale puisque ce faisant les Pygmées deviennent semblables à tous les paysans. Cette agriculture doit d'ailleurs s'accompagner de plantations de rente (cacao, café). C'est dans cet esprit que le Zaïre entama sa campagne de réhabilitation, libération et intégration des " premiers citoyens » (les Pygmées) ; c'est aussi ce principe qui guida la création dans les années 70 des villages-pilotes tels que ceux de haute Sangha (RCA) ou de l'arrondissement de Lomié (Cameroun).

Ces villages, nés de l'initiative de missionnaires catholiques, regroupent de très nombreux Pygmées (700 Baka à Moangue-Le Bosquet dans l'est du Cameroun, plus de $1000 \mathrm{Aka}$ à Belemboke dans le sud-ouest de la RCA). Des animateurs ruraux aidèrent les Pygmées à installer un habitat de type villageois, créèrent un dispensaire et une école, et travaillèrent au développement des cultures vivrières. Belemboke eut un succès certain et son attrait fut grand car il fut rapidement divisé en plusieurs villages, tout en drainant des campements spontanés en dehors de l'habitat-pilote (il y a maintenant près de 4000 Pygmées à 15 kilomètres à la ronde). A l'inverse, dans l'est du Cameroun, on note dans les années 80 une certaine tendance à un reflux vers la forêt.

A l'opposé de ces entreprises d'envergure se situent des actions fondées sur " l'alternative ». Elles combinent l'implantation d'un pôle d'attraction (d'abord dispensaire puis école) avec une animation itinérante menée directement dans les campements par les missionnaires (religieux et religieuses catholiques). C'est le cas dans la région de Bipindi (sud-ouest du Cameroun) ou de Moloundou (est du Cameroun) ou en Lobaye (RCA). Cependant, dans des régions où les villageois sont eux-mêmes défavorisés du point de vue médical, l'implantation en forêt d'une action destinée prioritairement aux Pygmées heurte les habitudes et peut provoquer des conflits entre les deux communautés.

La scolarisation des Pygmées est abordée généralement en accueillant les enfants dans les écoles rurales avec les villageois, l'ouverture d'écoles réservées aux jeunes Pygmées étant apparemment l'exception. Le principe est que le mélange des enfants travaille en faveur d'une connaissance mutuelle et d'une intégration progressive. Cependant, les difficultés rencontrées par les enfants pygmées pour suivre le cycle scolaire (éloignement des campements, timidité face aux villageois, analphabétisme des parents) a engagé certains missionnaires à assurer un encadrement pendant la scolarité (maison d'accueil des jeunes Pygmées à la mission catholique de Bipindi) ou même à créer une préscolarisation en baka des enfants (mission de Salapoumbé, est du Cameroun). Ail- 
leurs, on connaît des expériences malheureuses comme l'école pilote des Pygmées de Mongoumba (RCA) où malgré un grand succès l'année de l'ouverture, on ne trouvait plus, après deux ans, que des villageois.

Même dans les régions où les Pygmées sont effectivement demandeurs comme chez les Kola (Cameroun), la scolarisation se heurte partout au même obstacle, la mobilité. En effet, dans l'est (arrondissement de Moloundou) par exemple, il ne reste plus en fin d'année que $20 \%$ des Pygmées inscrits au début. On remarque aussi une désaffection qui se traduit par un taux d'inscription moindre d'année en année, cependant que l'on note une diminution de l'ordre du tiers entre 1980 et 1985 .

Rappelons, pour terminer, que l'ensemble de ces actions ne touche qu'un nombre extrêmement faible de Pygmées et que la grande majorité d'entre eux, bien que touché par l'économie moderne (comme on l'a détaillé précédemment), reste en dehors des projets.

\section{UN AVENIR ?}

Il y a maintenant plus de vingt ans que les pouvoirs publics ou les fondations privées tentent diverses actions auprès des chasseurs-collecteurs pygmées, et force est de constater l'échec de ces programmes, à tous les niveaux. Les villages-pilotes soit ont été en partie abandonnés (dans l'arrondissement de Lomié, le village passe de 700 en 1978 à 450 en 1984), soit continuent d'être des pôles problématiques où l'assistance technique est quotidiennement nécessaire. Les écoles comptent toujours aussi peu de Pygmées, et on cherchera sans succès des Pygmées ayant terminé un cycle scolaire. Et, paradoxalement, la grande masse des Pygmées qui n'a pas été prise en charge dans les projets de développement s'est néanmoins engagée, seule, sur le chemin des transformations. Ces échecs tiennent à trois facteurs : la difficulté de vivre en trop larges communautés, le peu d'intérêt pour l'agriculture et le profond attachement à une mobilité tournée vers la symbiose avec la forêt.

En analysant l'ensemble des situations observées chez les Pygmées, on en déduit quelques constantes. En premier lieu vient la passion de la chasse : même les salariés continuent à chasser. En second lieu, et étroitement lié, vient la mobilité saisonnière : rares sont les Pygmées qui ne partent pas au moins pour quelques semaines, afin de camper en forêt pour chasser ou récolter. La durée des voyages en forêt varie de deux à six mois même dans les villages permanents. Le mode de vie pygmée est toujours nettement marqué par une dualité qui perdure : vie en forêt alternant avec vie au village. En dernier lieu s'affirme une attitude vis-à-vis du salaire (ou du travail salarié) qui correspond à un approvisionnement à court terme : travailler le temps nécessaire à la satisfaction d'un besoin précis. 


\section{Un nouvel état d'esprit}

Certains pays, sans même envisager des programmes spécifiques de développement concernant les Pygmées, se laissent aller à la "tentation touristique $»^{5}$, et considèrent leurs Pygmées comme un patrimoine, au même titre que les réserves de faune et les chutes d'eau spectaculaires, laissant les agences de voyage organiser publicité et excursions dans les forêts les plus proches de la capitale.

Cette attitude reste heureusement limitée. Les praticiens de terrain comme les responsables politiques et administratifs reconnaissent maintenant les résultats limités des projets classiques, et peu à peu un nouvel état d'esprit se fait jour, où l'on abandonnerait l'idée universelle des villages-pilotes d'intégration, pour des campagnes plus subtiles. Les gouvernements les plus éclairés comme celui du Cameroun ont en effet engagé des recherches sociologiques (Institut des Sciences humaines pour le ministère des Affaires sociales) qui seraient préliminaires à des projets d'intégration. Leurs jeunes chercheurs s'interrogent sur le bienfondé du développement, et certains font le parallèle entre le passé colonial infligé à leurs pères, et ce que l'État moderne inflige aux Pygmées : " Le développement pygmée doit-il passer nécessairement par les étapes que les ex-colonisés ont subies? " (Ngima-Mawoung 1981).

\section{Une culture pygmée}

L'attitude générale de la majorité des Pygmées, qu'on interprète facilement comme un manque de persévérance ou un assujettissement au poids de la tradition, doit en fait être interprétée comme un refus.

Les Pygmées se comportent actuellement vis-à-vis de l'administration ou des projets de développement, comme ils se sont toujours comportés vis-à-vis de leurs patrons villageois : un acquiescement de façade, quelque chose que l'on pourrait appeler "le oui du refus" : dire oui et ne rien faire, sinon se sauver. Il s'agit là d'une réelle stratégie, même si nul leader ne l'organise.

Les Pygmées nous présentent l'image paradoxale d'une culture étonnamment puissante malgré son apparente fragilité. On ne peut qu'être admiratif devant la persistance de ces hommes dans leur mode de vie, à travers les siècles, en dépit des influences de l'histoire dont leurs langues gardent les traces. Les langues des Pygmées, même si elles sont différentes d'un pays à l'autre, sont aussi la marque d'une culture homogène : les locuteurs pygmées, aka ou baka par exemple, sont plus nombreux et couvrent des surfaces considérablement plus vastes que n'importe quelle langue de villageois qui leur est apparentée (plus de $10000 \mathrm{Aka}$ sur près de 100000 kilomètres carrés, contre moins

5. Cf. par exemple le Plan de mise en valeur du territoire de la RCA (1968):

"Potentiel touristique : la RCA possède sur un vaste territoire de nombreuses curiosités d'un intérêt certain :

- les chutes de Boali, Lancrenon, Kembé, etc. ;

- certaines régions : la forêt et les Babingas, les bords de l'Oubangui, des montagnes comme le Djebel Mella ;

- enfin et surtout, la présence d'une faune nombreuse et variée dans le nord-est et l'est du pays. " 
de 3000 Ngando sur 2500 kilomètres carrés, plus de 15000 Baka sur près de 70000 kilomètres carrés contre moins de 2000 Ngbaka sur 5000 kilomètres carrés!).

Qu'il n'y ait actuellement nulle part d'organisation ethnique pygmée ne signifie nullement que ceux-ci n'ont pas de conscience ethnique. L'absence d'organisation est aussi un trait de culture correspondant exactement à la fonction de responsabilité qui est le moteur des groupes sociaux pygmées : chaque individu est responsable et solidaire de tous les autres, matériellement et moralement. Cet " individualisme de groupe » est tel que le libre choix de chacun est respecté par les autres, même pour les enfants : dans l'est du Cameroun, les parents baka ne peuvent pas obliger leurs enfants à aller à l'école s'ils n'en ont pas envie.

Qu'il n'y ait nulle organisation ethnique ne signifie pas manque de conscience de différence. On entend d'ailleurs quelquefois des Pygmées utiliser pour se caractériser vis-à-vis des villageois, les termes de " Babinga ", de " pays ", voire de « Piguimé » qui leur sont normalement étrangers. Mais la mesure de leur infériorité civile dans le contexte moderne ne leur échappe pas ; ainsi la raison principale, avouée, de l'installation des Aka dans le village de Belemboke est-elle d'aller faire des champs " pour être comme les villageois ", c'està-dire pour être reconnus citoyens. Ailleurs (Ituri), on a vu des Mbuti emprunter de l'argent à leur patron et aller le porter au bourg pour payer l'impôt et obtenir ainsi un papier officiel.

On trouve là, exprimés par ces comportements, les deux aspirations profondes de nombreux Pygmées : devenir des citoyens à part entière, sans cesser d'être Pygmées ; et affirmer l'attachement à leur culture (langue, organisation sociale, choix techniques et culturels) tout en accédant aux mêmes droits que les autres (état civil, justice, santé).

La voie à suivre est délicate mais nette. Prenant acte des échecs passés, il est temps de s'écarter définitivement des projets directifs qui sont à la fois coûteux et inefficaces.

Il convient de faciliter l'accès des Pygmées à l'état civil, et en même temps mettre à leur portée une scolarisation adaptée à la fois à leurs besoins et à leur mode de vie. Il est bien évident qu'une meilleure intégration dans la société moderne passe par l'alphabétisation et la connaissance élémentaire du calcul. Les obstacles rencontrés par les enfants pygmées (timidité à cause de leur minorité) disparaîtront à mesure qu'ils seront plus nombreux à aller à l'école, de même que le phénomène de rejet (de la part des deux sociétés, celle des Pygmées comme celle des villageois) auquel sont en butte les rares Pygmées diplômés. L'adaptation principale consisterait à créer un cycle d'études en accord avec les rythmes de migration et qui, de ce fait, limiterait les absences des enfants en fin d'année scolaire. Le second point qui semble bénéfique est une préscolarisation en langue pygmée, qui familiariserait les enfants avec les principes de l'écriture et de la lecture sans entraîner les dégâts que provoque toujours l'immersion brutale dans le système de représentations d'une autre civilisation.

La « libération » des Pygmées, c'est-à-dire la séparation d'avec les villageois, ordinairement posée comme préliminaire à une politique d'intégration, 
n'est pas aisée à mener à bien ; elle entraîne même des conséquences généralement imprévues. L'économie rurale a été fondée par les villageois en tenant compte de la force de travail de leurs clients Pygmées (tout particulièrement en ce qui concerne les superficies cultivées, même en café et cacao). La suppression de cette main-d'œuvre entraîne souvent une incapacité pour le planteur d'entretenir la totalité de ses cultures, et l'oblige à en abandonner une partie.

L'amélioration du sort des Pygmées passe nécessairement par une éducation des villageois, renforcée d'ailleurs par une juste application de la réglementation du travail en vigueur.

La volonté affirmée des pouvoirs publics de « faire participer les Pygmées à l'effort de construction nationale " sous-entend que ceux-ci restent actuellement en dehors de la vie économique de l'État. On a vu plus haut qu'il n'en était rien, et qu'actuellement comme autrefois les Pygmées sont pleinement intégrés dans des circuits économiques régionaux. De nombreux citadins ou ouvriers mangent, sans le savoir peut-être, de la viande provenant des chasses pygmées. Mais, ce faisant, les Pygmées ne correspondent pas au schéma habituel du monde rural : on considère que les Pygmées, ne cultivant pas, ne sont pas des paysans comme les autres, et de ce fait représentent une force de travail qui fait défaut pour le développement de régions sous-peuplées. Il est nécessaire et légitime d'encourager les Pygmées qui souhaitent devenir agriculteurs. Mais il y a aussi une place pour tous les autres. Dans les régions forestières, les chasseurs professionnels pygmées ont une fonction cruciale pour l'approvisionnement.

En fait, le sort des Pygmées dépend étroitement du devenir de la forêt équatoriale (ce qui a déjà été maintes fois proclamé) et l'on doit envisager les problèmes de développement de ces régions à un niveau beaucoup plus global. Dans cet écosystème vulnérable par excellence, et très rapidement perturbé dès lors qu'il est abusivement exploité, l'interdépendance des hommes et du milieu est une donnée fondamentale. Les États seront rapidement confrontés à de graves problèmes écologiques, et le développement de ces régions dépendra de la manière dont ils seront abordés et des solutions qui y seront apportées.

En effet, de nombreux problèmes conjoints se posent : enrichissement et valorisation des forêts après une première exploitation, protection des sols contre l'érosion, compétition entre maintien d'un couvert forestier ou mise en agriculture, conservation de la faune et de la flore et également paupérisation des paysans dans les zones exploitées. Les ministères compétents ont d'ores et déjà à planifier l'après-exploitation, à programmer des reboisements, à prévoir la mise en réserve de certaines des zones. Cependant les organismes internationaux (FAO, Unesco et même Banque mondiale) s'intéressent déjà à d'autres solutions, à d'autres conceptions, où l'on reconnaît l'importance écologique des couverts forestiers et les apports essentiels à l'équilibre régional (maintien hydraulique, protection contre les sécheresses), ainsi que l'existence de produits secondaires autres que le bois d'œuvre ou la cellulose : diversité des ressources génétiques, aliments végétaux et animaux, produits pharmaceutiques, miel...

L'agroforesterie apparaît comme l'une des meilleures solutions d'avenir, puisqu'elle vise par une agro-sylviculture soigneusement étudiée au niveau régional, à domestiquer la forêt en la mêlant aux systèmes agraires, plutôt qu'à la faire disparaître. Les mêmes organismes mettent désormais en garde contre les 
préjudices que les projets de développement apportent aux « groupes tribaux » (Tribals people) et invitent à prendre en compte leur survie et leur identité ethnique (Goodland 1982). C'est également dans la perspective d'une planification plus écologique de l'utilisation du milieu forestier que ces mêmes communautés peuvent intervenir, globalement, par l'apport de leur immense savoir ancestral en matière d'écologie forestière, et pratiquement, en utilisant pour cette nouvelle exploitation leurs talents de forestiers professionnels (nul mieux qu'un Pygmée ne peut localiser les plantes médicinales ou les arbres à miel dans l'immensité de la forêt). On peut imaginer ainsi de les employer en priorité et en exclusivité comme prospecteurs, récolteurs de produits sauvages, voire comme chasseurs professionnels (après réglementation de la chasse et gestion des populations animales) ou captureurs d'animaux pour les parcs zoologiques, ou encore comme guides, pisteurs et gestionnaires de réserves de faune et de flore.

Dans une perspective d'exploitation et d'aménagement harmonieux, d' " anthropisation » de l'écosystème forestier, les forestiers professionnels que sont les Pygmées, pourvus d'un savoir ancestral inestimable, ont un grand rôle à jouer. Puissent-ils redevenir pour les États modernes ce qu'ils furent pour les premiers Grands Noirs : les guides de la forêt !

Paris, CNRS, Lacito

\section{BIBLIOGRAPHIE}

Althabe, G. 1965. "Changements sociaux chez les Pygmées Baka de l'EstCameroun ", Cahiers d'études africaines, V - 20 : 561-92.

AROM, S. \& J.M.C. THOMAS, 1974. Les Mimbo, génies du piégeage et le monde surnaturel des Ngbaka-ma'bo (RCA). Paris, Selaf.

BAHUCHET, S. 1972. "Étude écologique d'un campement de Pygmées BaBinga (République centrafricaine) ", Journal d'Agriculture tropicale et de botanique appliquée, XIX - 12 : 509-59.

- 1979. «Utilisation de l'espace forestier par les Pygmées Aka, chasseurs-cueilleurs d'Afrique centrale », Social Sciences Information, 18-6 : 999-1 019.

- 1984. "Circulation et échanges en Afrique tropicale : relations entre chasseurscueilleurs pygmées et agriculteurs de forêt en Centrafrique ", Revista de Préhistoria, VI : 86-97.

- 1985. Les Pygmées Aka et la forêt centrafricaine, ethnologie écologique. Paris, Selaf.

- 1987. "Le filet de chasse des Pygmées Aka (RCA) ", in Koechlin, Sigaut, et al., De la voûte céleste au terroir, du jardin au foyer : mosaïque sociographique. Paris, EHESS : 209-26.

- 1988. "Food supply uncertainty among the Aka Pygmies (Lobaye, CAR) », in I. de Garine \& G.A. Harrison, Coping with uncertainty in food supply. Oxford, Oxford University Press : 118-49.

- 1989. Les Pygmées Aka et Baka : contribution de l'ethnolinguistique à l'histoire des populations forestières d'Afrique centrale. Université René DescartesParis $\mathrm{V}$, thèse de doctorat d'État es lettres et sciences humaines. 
BAHUCHET, S. \& H. GUILlAUME, 1979. "Relations entre chasseurs-collecteurs Pygmées et agriculteurs de la forêt du nord-ouest du bassin congolais ", in S. Bahuchet, Pygmées de Centrafrique. Paris, Selaf : 109-39.

- 1982. "Aka-farmer relations in the northwest Congo basin ", in E. Leacock \& R.B. Lee, Politics and history in band societies. Cambridge/Paris, Cambridge University Press /MSH : 189-211.

BAHUCHET, S. \& J.M.C. THOMAS, 1985. « Conservation des ressources alimentaires en forêt tropicale humide : chasseurs-cueilleurs et proto-agriculteurs d'Afrique centrale ", in Les techniques de conservation des grains à long terme. Paris, Éditions du CNRS : 15-31.

- 1986. "Linguistique et histoire des Pygmées de l'ouest du bassin congolais ", Sprache und Geschichte in Afrika, 7-2: 73-103.

- 1987. "Pygmy Religions", in M. Eliade, The Encyclopedia of Religion. New York, McMillan : 107-110.

BAILEY, R.C. 1982. " Development in the Ituri forest of Zaïre », Cultural Survival Quarterly, $6: 23-25$.

BAILEY, R.C. \& N.R. PEACOCK, 1988. Efe Pygmies of northeast Zaïre : subsistence strategies in the Ituri forest. In I. de Garine \& G.A. Harrison, Coping with uncertainty in food supply. Oxford, Oxford University Press : 88-117.

BRISSON, R. 1981-84. Contes des Pygmées Baka du Sud-Cameroun; vol. 1 et 2, Histoires et contes d'enfants; vol. 3 et 4, Contes des anciens. Douala, BP 5351.

COQUERY-VIDROVITCH, C. \& H. MONIOT, 1974. L'Afrique noire, de 1800 à nos jours. Paris, Presses universitaires de France.

COUdrin, H. 1988. Les Pygmées Mbenzele en quête de la maîtrise de leur devenir ; analyse d'une mutation sociale à partir de l'auto-évaluation d'un projet de développement. Strasbourg II, Diplôme des Hautes Études de la pratique sociale.

DELOBEAU, J.M. 1978. "Yamonzombo et Yandenga : histoire des relations entre les gens du fleuve et les gens de la forêt (XIXe-XXe siècle), Cahiers congolais, 2-3: 43-55.

- 1989. Yandenga et Yamonzombo. Les relations entre les villages monzombo et les campements pygmées aka dans la sous-préfecture de Mongoumba (Centrafrique). Paris, Peeters-Selaf.

DEMESSE, L. 1978. Changements techno-économiques et sociaux chez les Pygmées Babinga (nord-Congo et sud-Centrafrique). Paris, Selaf.

- 1980. Techniques et économie des Pygmées Babinga. Paris, Institut d'ethnologie.

GoOdLAND, R. 1982. Tribal peoples and economic development. Human ecologic considerations. Washington, World Bank.

HARAKO, R. 1976. "The Mbuti as hunters : as study of ecological anthropology of the Mbuti Pygmies (I) », Kyoto University African Studies, X : 37-99.

HART, J.A. 1978. « From subsistance to market : a case study of the Mbuti net hunters ", Human Ecology, VI-3 : 325-53.

HART, J.A. \& T.B. HART, 1984. " The Mbuti of Zaïre. Political change and the opening of the Ituri forest ", Cultural Survival Quarterly, 8-3 : 18-20.

ICHIKAWA, M. 1978. "The residential groups of the Mbuti Pygmies ", Senri Ethnological Studies, 1-Africa 1 : 131-88.

- 1981. "Ecological and sociological importance of honey to the Mbuti net hunters, Eastern Zaïre ", African Study Monographs, 1 : 55-68.

KAZADI, M. 1981. "Méprisés et admirés : l'ambivalence des relations entre les Bacwa (Pygmées) et les Bahemba (Bantu) », Africa, 51-4 : 837-47. 
KEIM, C.A. 1983. "Long-distance trade and the Mangbetu ", Journal of African History, $24: 1-22$.

LOUNG, J.F. 1959. « Les Pygmées de la forêt de Mill. Un groupe de Pygmées camerounais en voie de sédentarisation ", Les Cahiers d'outre-mer, 48 : 362-79.

NGUima-Mawoung, G. 1981. L'intégration des Pygmées dans la société camerounaise ; le cas du secteur de Bipindi dans le département de l'Océan. Université de Yaoundé, mémoire de maîtrise de sociologie.

PAGEZY, H. 1982. "Seasonal hunger, as experienced by the Oto and the Twa of a Ntomba village in the equatorial forest ", Ecology of Food and Nutrition, $12: 139-53$.

- 1986. "The food system of the Ntomba of Lake Tumba, Zaïre », in : J. Pottier, Food systems in central and southern Africa. London, SOAS.

PEACOCK, N. 1984. "The Mbuti of northeast Zaïre. Women and subsistence exchange ", Cultural Survival Quarterly, 8-2 : 15-17.

SCHebesta, P. 1952. Les Pygmées du Congo belge. Bruxelles, Mémoires de l'Institut royal colonial belge, XXVI-2.

SCHULTZ, M. 1986. "Economic relations between the Batua and Baoto of Bibelo Village, Bikoro zone, Zaïre : a preliminary report on new fieldwork ", Sprache und Geschichte in Afrika, 7-2: 339-59.

SÉVY, G. 1972. Terre ngbaka. Évolution de la culture matérielle d'une population rurale forestière de République centrafricaine. Paris, Selaf.

SUlzMANN, E. 1986. " Batwa und Baoto - die Symbiose von Wildbeutern und Pflanzern bei den Ekonda und Bolia (Zaïre) ", Sprache und Geschichte in Afrika, 7-1: 369-89.

SURET-CANALE, J. 1977. Afrique noire occidentale et centrale; l'ère coloniale (1900-1945). Paris, Éditions sociales.

THOMAS, J.M.C. 1963. Les Ngbaka de la Lobaye. Le dépeuplement rural chez une population forestière de la République centrafricaine. Paris, Mouton.

- 1979. «Emprunt ou parenté ? A propos des parlers des populations forestières de Centrafrique ", in S. Bahuchet, Pygmées de Centrafrique. Paris, Selaf : 141-69.

TURnBUll, C.M. 1965. Wayward servants, the two worlds of the African Pygmies. New York, The Natural History Press.

- 1968. "The importance of flux in two hunting societies ", in R.B. Lee \& I. De Vore, Man the Hunter. Chicago, Aldine : 132-37.

- 1983. The Mbuti Pygmies; change and adaptation. New York, Holt, Rinehart \& Winston.

VAllois, H.V. \& P. MARQueR, 1976. Les Pygmées Baka du Cameroun : anthropologie et ethnographie. Paris, Muséum national d'histoire naturelle.

WAEHLE, E. 1986. «Efe (Mbuti Pygmy) relations to Lese-Dese villagers in the Ituri forest, Zaïre : historical changes during the last 150 years ", Sprache und Geschichte in Afrika, 7-2: 375-411. 


\title{
LES PYGMÉES D’AUJOURD’HUI EN AFRIQUE CENTRALE SERGE BAHUCHET
}

\begin{abstract}
A la suite d'une description rapide du mode de vie traditionnel des Pygmées d'Afrique centrale, les transformations actuelles des forêts de cette région sont examinées. En dépit de la dispersion des Pygmées et malgré les différences entre ces populations, les mêmes types d'évolution du mode de vie se trouvent partout. L'auteur cherche à dégager les racines historiques et sociales de ces tendances en se référant à l'histoire des relations entre Pygmées et villageois et en examinant le contexte économique actuel des forêts d'Afrique équatoriale. Une présentation de cas socio-économiques est suivie de l'exposé des principaux problèmes sociaux, des politiques de développement et des questions (et suggestions) pour l'avenir.
\end{abstract}

\section{PYGMIES TODAY IN CENTRAL AFRICA SERGE BAHUCHET}

\begin{abstract}
After a brief description of central African Pygmies' traditional way of life, the current transformations of the region's rain forests are pointed out. Despite the dispersion of, and differences between, these populations, life-style patterns are evolving in like manner everywhere. The social and historical roots of these trends are examined, in particular the economic context of the African rain forests and the history of Pygmy-farmer relations. The presentation of contemporary socioeconomic cases is followed by a discussion of development policies and major social problems. Questions are raised, and suggestions made, about the Pygmies' future.
\end{abstract}

